2. Weick JK, Kopecky KJ, Appelbaum FR, et al. A randomized investigation of high-dose versus standard-dose cytosine arabinoside with daunorubicin in patients with previously untreated acute myeloid leukemia: a Southwest Oncology Group study. Blood 1996;88:2841-51.

3. Bradstock KF, Matthews JP, Lowenthal RM, et al. A randomized trial of high-versus conventional-dose cytarabine in consolidation chemotherapy for adult de novo acute myeloid leukemia in first remission after induction therapy containing high-dose cytarabine. Blood 2005;105:481-8.
4. Büchner T, Berdel WE, Schoch $\mathrm{C}$, et al. Double induction containing either two courses or one course of high-dose cytarabine plus mitoxantrone and postremission therapy by either autologous stem-cell transplantation or by prolonged maintenance for acute myeloid leukemia. J Clin Oncol 2006;24:2480-9. 5. Moore JO, George SL, Dodge RK, et al. Sequential multiagent chemotherapy is not superior to high-dose cytarabine alone as postremission intensification therapy for acute myeloid leukemia in adults under 60 years of age: Cancer and Leukemia Group B Study 9222. Blood 2005;105:3420-7.

\title{
The ClinicalTrials.gov Results Database
}

TO THE EDITOR: Zarin et al. (March 3 issue) ${ }^{1}$ provide an update on ClinicalTrials.gov, currently the only publicly available database reporting trial results; it should be used in systematic reviews and meta-analyses. Of the researchers who wrote more than 12,000 such reviews and meta-analyses published and indexed in PubMed between 2009 and 2010, less than 2\% reported using ClinicalTrials.gov to search for unpublished data. ${ }^{2}$ The scarce utilization suggests the scientific community is not sufficiently aware of ClinicalTrials .gov or else struggles with implementation of this key resource for synthesis of research.

Moritz Wyler von Ballmoos, M.D., M.P.H.

Medical College of Wisconsin

Milwaukee, WI

mcwvb@post.harvard.edu

Carol Oliveira, M.D.

University of Toronto

Toronto, ON, Canada

No potential conflict of interest relevant to this letter was reported.

1. Zarin DA, Tse T, Williams RJ, Califf RM, Ide NC. The ClinicalTrials.gov results database - update and key issues. N Engl J Med 2011;364:852-60.

2. Montori VM, Wilczynski NL, Morgan D, Haynes RB. Optimal search strategies for retrieving systematic reviews from Medline: analytical survey. Br Med J 2005;330:68.

TO THE EDITOR: We report an experience with ClinicalTrials.gov and our international randomized, controlled trial CHIPS (Control of Hypertension In Pregnancy Study). CHIPS is funded by the Canadian Institutes of Health Research, which mandates registration and assignment of an International Standard Randomised Controlled Trial Number (ISRCTN) (no. 71416914). However, one of our active international sites discovered that CHIPS had been registered on ClinicalTrials.gov by another interested (but not active) site. The
ClinicalTrials.gov entry contained many factual errors. Although we have now overridden this submission, is there a mechanism to prevent duplicate and inaccurate registration?

Laura A. Magee, M.D.

Jennifer Menzies, M.Sc.

University of British Columbia

Vancouver, BC, Canada

Imagee@cw.bc.ca

\section{for the CHIPS Study Group}

No potential conflict of interest relevant to this letter was reported.

TO THE EDITOR: There are considerable disincentives to the reporting requirement in ClinicalTrials .gov. Three examples include the fact that all data entry has to be performed manually rather than being uploaded with the use of commonly used software programs and formats such as Microsoft Word, Adobe Portable Document Format (PDF), Microsoft Excel, or SAS statistical software. Another problem is that the different measures of "dispersion" (e.g., standard error, standard deviation, 95\% confidence interval, and interquartile range) within studies add complexity to data entry in various tables. Finally, the space allotted to specific sections (e.g., statistical analysis) is insufficient.

\section{Michael Camilleri, M.D.}

Mayo Clinic

Rochester, MN

camilleri.michael@mayo.edu

No potential conflict of interest relevant to this letter was reported.

TO THE EDITOR: ClinicalTrials.gov has important quality-control measures to prevent information in registered records from being omitted, incomplete, incorrect, or uninformative. Unfortunately, 
the quality of registered trial data across registries remains a problem. Using one of the criteria proposed by Zarin et al., we identified "internal inconsistency" as a problem in about $9 \%$ of records in the World Health Organization (WHO) International Clinical Trials Registry Platform (ICTRP) search portal. ${ }^{1}$ In the records for these trials, multiple descriptors that are not compatible - such as "single-group" and "controlled or randomized"; "open-label" and "blinded"; and "double-blinded without subject or investigator blinding" - were used.

The ICTRP has now established International Standards for Clinical Trial Registries, which set a minimum requirement for quality-control processes performed and data recording practices used by individual registries. The intention is to implement these minimum standards and monitor compliance by registries in the WHO Registry Network to ultimately achieve the recording of more complete and meaningful clinical-trial information.

Roderik F. Viergever, M.D.

London School of Hygiene and Tropical Medicine London, United Kingdom

Davina Ghersi, M.P.H., Ph.D.

World Health Organization

Geneva, Switzerland

ghersid@who.int

Dr. Ghersi reports being the team leader of the International Clinical Trials Registry Platform of the World Health Organization. No other potential conflict of interest relevant to this letter was reported.

1. Viergever RF, Ghersi D. The quality of registration of clinical trials. PLoS ONE 2011;6(2):e14701.

THE AUTHORS REPLY: We agree with Wyler von Ballmoos and Oliveira that ClinicalTrials.gov is an important resource for systematic reviewers as well as for anybody else (e.g., members of institutional review boards) who seeks the complete evidence base on a given topic. Since the results of many clinical trials remain unpublished, ${ }^{1}$ even years after completion, it is clear that a literature search alone will not result in a complete picture of the evidence.

Magee and Menzies raise the issue of duplicate and inaccurate registrations. We previously addressed the importance of preventing duplicate registrations and ensuring the accuracy of reg- istrations. ${ }^{2}$ To prevent unintended duplicate registrations, the sponsor, study sites, and all relevant parties must identify the one person who will be responsible for trial-registration and related reporting activities; that person would be designated as the responsible party in ClinicalTrials.gov. Individual study-site investigators should not register their study unless they serve as the responsible party for the overall trial. If a study must be submitted to multiple registries (e.g., to comply with regional policies), we ask that the NCT number and other registry identifiers be listed in all registrations to allow for the identification of (intentional) duplicate records. Regarding inaccuracies, although our staff manually reviews each record, certain factual errors cannot be detected without access to the study data. It is essential that the responsible party has the necessary expertise to submit accurate and informative data.

Camilleri notes that ClinicalTrials.gov does not allow for the uploading of data in formats used by popular software platforms. However, ClinicalTrials.gov does support the uploading of data in the Extensible Markup Language (XML) format, a standard method of encoding information in an unambiguous machine-readable structure - unlike the data-file formats mentioned by Camilleri. Without the structured numeric entries afforded by the XML format, it would not be possible for the ClinicalTrials.gov system to perform automated validation of entries or to provide consistent displays of data.

We agree with Viergever and Ghersi that registries must implement quality-control measures. However, the quality of entries will always be dependent on the diligence and integrity of the person entering the data.

\section{Tony Tse, Ph.D. \\ Nicholas C. Ide, M.S. \\ Deborah A. Zarin, M.D.}

National Library of Medicine

Bethesda, MD

dzarin@mail.nih.gov

Since publication of their article, the authors report no further potential conflict of interest.

1. Ross JS, Mulvey GK, Hines EM, Nissen SE, Krumholz HM. Trial publication after registration in ClinicalTrials.gov: a crosssectional analysis. PLoS Med 2009;6(9):e1000144.

2. Zarin DA, Ide NC, Tse T, Harlan WR, West JC, Lindberg DAB. Issues in the registration of clinical trials. JAMA 2007;297:211220. 